\title{
Synergistic effects of granulocyte-colony stimulating factor and macrophage-colony stimulating factor on recovery of donor hematopoietic cells in allogeneic bone marrow transplantation
}

\author{
YUMING ZHANG $^{1,5}$, YASUSHI ADACHI ${ }^{1-3}$, YASUHIRO SUZUKI ${ }^{1}$, KEIZO MINAMINO $^{1}$, \\ MASAYOSHI IWASAKI ${ }^{1}$, KEIJI NAKANO ${ }^{1}$, YASUSHI KOIKE ${ }^{1}$, HIROMI MUKAIDE $^{1}$, \\ AKIO SHIGEMATSU, NAOKO KIRIYAMA ${ }^{1}$, CHUNFU LI ${ }^{5}$ and SUSUMU IKEHARA ${ }^{1-4}$ \\ ${ }^{1}$ First Department of Pathology, ${ }^{2}$ Regeneration Research Center for Intractable Diseases, ${ }^{3}$ Center for \\ Cancer Therapy, ${ }^{4}$ Department of Transplantation for Regeneration Therapy, Kansai Medical University, \\ Moriguchi, Japan; ${ }^{5}$ Department of Pediatrics, Nanfang Hospital, Guangzhou, P.R. China
}

Received February 2, 2006; Accepted March 30, 2006

\begin{abstract}
G-CSF and M-CSF are used clinically to augment hematopoiesis after bone marrow transplantation (BMT) and chemotherapy. In this paper, we examined the synergistic effect of G-CSF and M-CSF on hematopoietic recovery in allogeneic BMT as a model of human BMT. We performed BMT from eGFP-transgenic mice (C57BL/6 background; $\mathrm{H}-2^{\mathrm{b}}$ ) into lethally-irradiated $\mathrm{C} 3 \mathrm{H}\left(\mathrm{H}-2^{\mathrm{k}}\right)$. From the day after BMT, G-CSF and/or M-CSF were injected for 5 consecutive days. Not only the numbers of day $12 \mathrm{CFU}-\mathrm{S}$ and spleen weight, but also white blood cell (WBC) counts in the peripheral blood (PB) and nuclear cells in the bone marrow (BM) increased in the mice treated with G-CSF and/or M-CSF 12 days after BMT. Moreover, the number of donor-type WBCs in the PB and donor-type nuclear cells in the $\mathrm{BM}$ also increased in the mice treated with G-CSF and/or M-CSF. The effects were pronounced when G-CSF and M-CSF were used together rather than independently. These results suggest that treatment with the combination of G-CSF and M-CSF has a synergistic effect on hematopoiesis in allogeneic BMT.
\end{abstract}

\section{Introduction}

A critical side effect of bone marrow transplantation (BMT) is infection due to a serious decrease in the number of WBCs.

Correspondence to: Dr Susumu Ikehara, First Department of Pathology, Kansai Medical University, 10-15 Fumizono-cho, Moriguchi city, Osaka 570-8506, Japan

E-mail: ikehara@takii.kmu.ac.jp

Key words: granulocyte-colony stimulating factor, macrophagecolony stimulating factor, colony stimulating unit of spleen, hematopoiesis
Therefore, the early recovery of transplanted donor hematopoietic cells is crucial for the prevention of infection. Several cytokines are clinically used to augment the number and function of white blood cells (WBCs); myeloid lineage cells, such as granulocyte colony stimulating factor (G-CSF), macrophage colony stimulating factor (M-CSF) and granulocyte-macrophage colony stimulating factor (GM-CSF) have been tried in myelodysplastic chemotherapy and BMT. G-CSF accelerates neutrophil recovery, reduces the total number of febrile days and reduces the frequency of infection in the treatment of acute myeloid leukemia (1). Although G-CSF augments not only the differentiation of bone marrow stem cells (BMSCs) into neutrophils but also proliferation of leukemia cells in vitro (2), clinical trials of G-CSF not only failed to show the expansion of leukemia cells, but also showed a higher remission rate in the treatment of AML (1). M-CSF not only promotes the proliferation and differentiation of progenitor cells of monocyte-macrophage lineage cells (3), but also has anti-tumor effects (4), supports pregnancy (5) and augments anti-fungal effects through activation of monocyte-macrophage lineage cells (6). M-CSF actually reduced the incidence and duration of febrile neutropenia during consolidation therapy in the AML patients (7). M-CSF failed to prolong disease-free survival but did not show any particular adverse effects.

In contrast to the two cytokines described above, the results of clinical trials of GM-CSF are controversial. The Eastern Cooperative Oncology Group (ECOG) showed that GM-CSF shortens the recovery time of neutrophils, and resulted in a reduction in the number of infections and a lower mortality rate in AML patients that received chemotherapy. They also showed that GM-CSF improved the remission rate (8). However, the Cancer and Leukemia Group B (CALGB) failed to show any improvements in mortality or remission rate with GM-CSF (9). The European Organization for Research and Treatment of Cancer (EORTC) showed that treatment with GM-CSF after chemotherapy accelerated hematopoiesis but reduced the remission rate of AML patients, probably due to 
acceleration of regrowth of residual leukemia cells (10). They concluded that the administration of GM-CSF in the treatment did not provide any clinical benefits.

These reports suggest that both G-CSF and M-CSF are safe and have effects on the recovery of hematopoiesis, even in clinical use. Therefore, in this experiment, we examined the synergistic effects of the combination of G-CSF and M-CSF on the recovery of hematopoiesis of donor hematopoietic cells in allogeneic BMT.

\section{Materials and methods}

Mice. $\mathrm{C} 3 \mathrm{H} / \mathrm{HeN}\left(\mathrm{C} 3 \mathrm{H}, \mathrm{H}-2^{\mathrm{k}}\right)$ mice were purchased from Japan SLC, Inc. (Hamamatsu, Japan). C57/BL6 mice carrying the eGFP transgene (eGFP+ $\mathrm{B} 6$ mice) were kindly donated by Dr H. Okabe (Osaka University, Osaka, Japan) (11). All of the mice were maintained in a pathogen-free environment in our laboratory.

Experimental protocols. The recipient mice ( $\mathrm{C} 3 \mathrm{H}$ mice) were exposed to $9.5 \mathrm{~Gy}$ of $\gamma$ irradiation from a ${ }^{137} \mathrm{Cs}$ source (Gammacell 40 Exactor; Nordion International Inc., Kanata, Ontario, Canada) 1 day before transplantation. BMCs $\left(1 \times 10^{5}\right)$ from $\mathrm{eGFP}^{+} \mathrm{B} 6$ mice were transplanted via the tail vein to $\mathrm{C} 3 \mathrm{H}$ mice on day 0 . From the day after the BMT, G-CSF (125 $\mu \mathrm{g} / \mathrm{kg}$; donated by Chugai Pharmaceutical Co., Ltd., Tokyo, Japan) and/or M-CSF (125 $\mu \mathrm{g} / \mathrm{kg}$; donated by Morinaga, Tokyo, Japan) were injected intraperitoneally for 5 consecutive days. As a control, saline was injected instead of cytokine(s).

For assay of colony forming unit of spleen (CFU-S assay), the recipient mice were sacrificed 12 days after BMT, and their spleens were removed, weighed and fixed in Bouin's solution. Visible surface colonies were counted 1 day after fixation.

Analyses of chimerism. For analyses of the PB and BM cells, 12 days after transplantation, the peripheral WBCs and the nuclear cells from tibia were counted and phenotyped for the analyses of recipient/donor cell ratios by flow cytometry. In brief, PB of the recipient mice was collected and stained with phycoerythrin (PE)-conjugated anti-CD45 mAb (BD Biosciences, San Jose, CA), followed by hemolysis using BD PharM Lyse (BD Biosciences PharMingen). The stained cells were analyzed using a FACScan (Becton-Dickinson, Mountain View, CA). Both $\mathrm{CD}^{4} 5^{+}$and $\mathrm{eGFP}^{+}$cells in $\mathrm{CD}^{4} 5^{+}$cells were considered as donor-derived nuclear cells. The numbers of donor hematopoietic cells were calculated with the ratios of donor-derived cells in the PB or BM and absolute numbers of WBCs in the PB or nuclear cells in the BM.

Statistical analyses. Significances were evaluated using the Student's t-test.

\section{Results}

G-CSF and/or M-CSF enhance the number of CFU-S and augment the weight of the spleen. The colony-forming unitspleen (CFU-S) assays devised by Till and McCulloch have been considered a means of measuring counts of hematopoietic stem cells or hematopoietic progenitors; especially,
A.

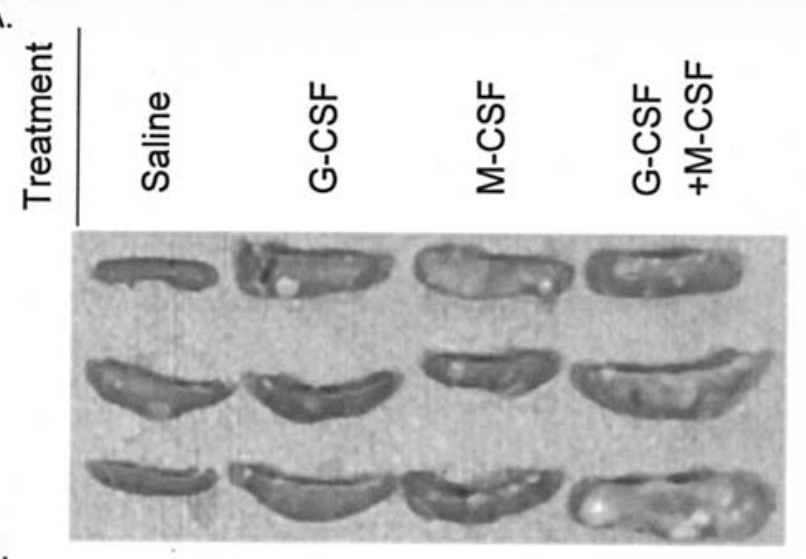

B.

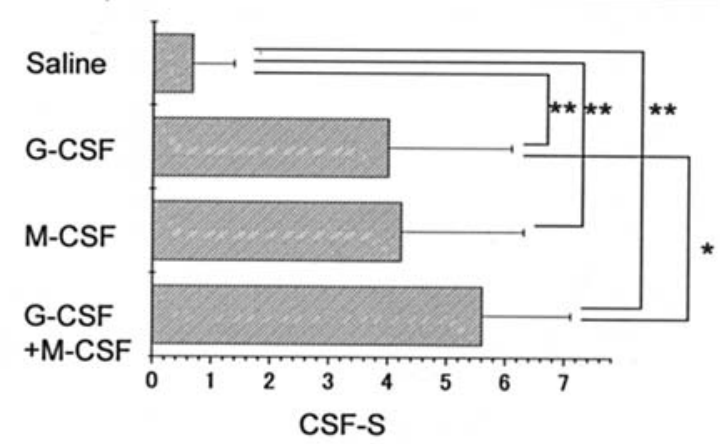

C.

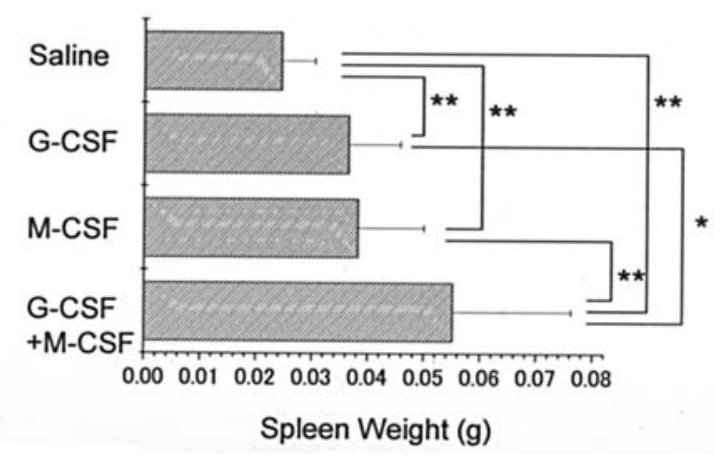

Figure 1. G-CSF and/or M-CSF accelerate hematopoiesis in allogeneic BMTBMCs $\left(1 \times 10^{5}\right)$ from eGFP+B6 mice were transplanted into lethally irradiated $\mathrm{C} 3 \mathrm{H}$ mice. Twelve days after BMT, mice were sacrificed and CFU-S and spleen weight were examined. (A), Shows representative data of CFU-S 12 days after BMT. (B and C) Show CFU-S and spleen weights, respectively. $n>6$ in each group.

CFU-S counts on day 12 (day 12 CFU-S) are thought to reflect the number of immature hematopoietic precursor and/or hematopoietic stem cells (12). Therefore, we first analyzed day 12 CFU-S and spleen weight to examine the effects of G-CSF and/or M-CSF on hematopoiesis in allogeneic BMT. From the day after transplantation, G-CSF and/or M-CSF were injected for 5 consecutive days and the mice were sacrificed on day 12. As shown in Fig. 1, both G-CSF-treatment and M-CSF-treatment are effective in augmenting CSF-S counts (Fig. 1A and B) and spleen weight (Fig. 1C) in comparison with saline-treatment. There was no significant difference in CSF-S counts between the mice treated with either G-CSF or M-CSF and the mice treated with both G-CSF and M-CSF, but the combination of G-CSF and M-CSF tended to be more effective. The mice treated with either G-CSF or M-CSF showed significantly heavier spleen weights than the mice treated with saline. The combination of G-CSF and M-CSF 
A.

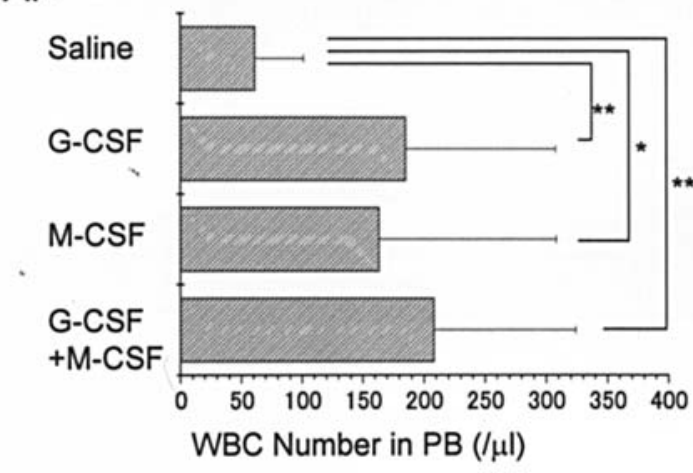

C.

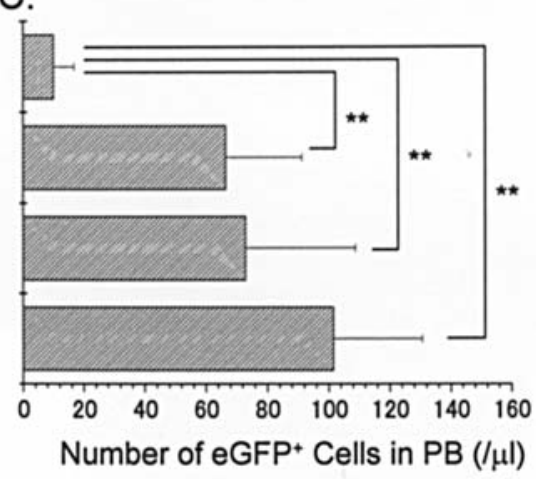

B.

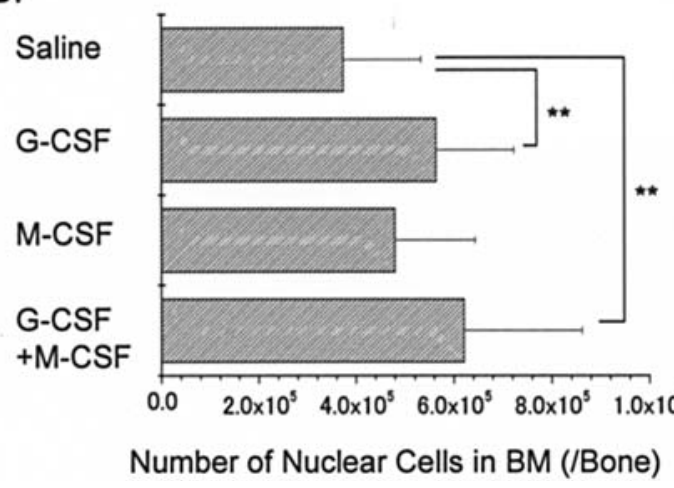

D.

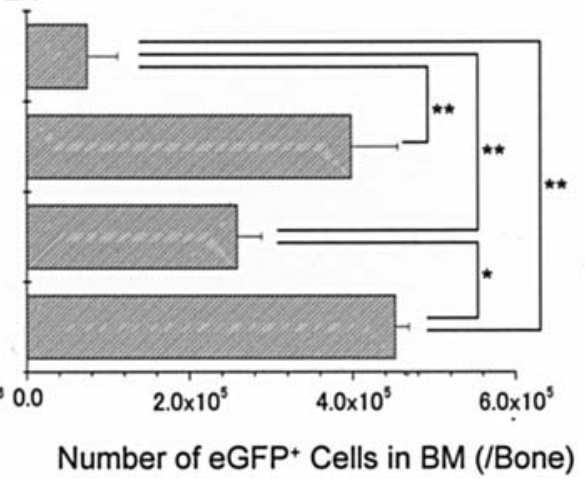

Figure 2. G-CSF and/or M-CSF augment donor derived nuclear cells in PB and BM in allogeneic BMT. BMCs $\left(1 \times 10^{5}\right)$ from eGFP+ $\mathrm{B} 6$ mice were transplanted into lethally-irradiated $\mathrm{C} 3 \mathrm{H}$ mice. Twelve days after BMT, WBC counts and the number of bone marrow cells in tibia were examined (A and B). The PB and the bone marrow cells were stained with PE-labeled anti-CD45 Ab and donor-derived cells, which were estimated with both eGFP+ and CD45+, were examined using a flow cytometer. The numbers of donor cells in the PB and BM were calculated with \% of donor cells and numbers of nuclear cells in the PB and BM. $n>6$ in each group.

treatment tended to be more effective than either M-CSFtreatment or G-CSF-treatment alone. These results suggest that G-CSF and M-CSF have effects on hematopoietic recovery in allogeneic BMT and that the combination of G-CSF and M-CSF treatment is more effective than either G-CSF or M-CSF treatment alone.

Effects of G-CSF and/or M-CSF on WBC counts in PB, nuclear cell counts in $B M$, and recovery of donor hematopoietic cells. In the $\mathrm{PB}$, total $\mathrm{WBC}$ counts and donor $\left(\mathrm{eGFP}^{+}\right)$ WBC counts in the mice treated with G-CSF and/or M-CSF increased significantly compared to mice treated with saline (Fig. 2A and C). There were no significant differences in WBC counts in the PB between the mice treated with G-CSF, the mice treated with M-CSF and the mice treated with a combination of G-CSF and M-CSF, but the combination of G-CSF and M-CSF was more effective than G-CSF or M-CSF treatment alone (Fig. 2A). Nuclear cells in the BM of mice treated with G-CSF and mice treated with a combination of G-CSF and M-CSF increased significantly in comparison with the mice treated with saline (Fig. 2B). There were no significant differences in numbers of nuclear cells in the BM between the mice treated with M-CSF and the mice treated with saline. Donor-derived BMCs were significantly increased in mice treated with G-CSF, mice treated with M-CSF, and mice treated with a combination of G-CSF and M-CSF (Fig. 2D). There were no significant differences in the numbers of nuclear cells in the BM and donor-derived hematopoietic cells in the BM between the mice treated with G-CSF, the mice treated with M-CSF and the mice treated with a combination of G-CSF and M-CSF, but the combination of G-CSF and $\mathrm{M}-\mathrm{CSF}$ was more effective than G-CSF or M-CSF alone.

\section{Discussion}

In this experiment, we showed the synergistic effects of G-CSF and M-CSF on hematopoiesis of donor hematopoietic cells in allogeneic BMT.

G-CSF, M-CSF and GM-CSF are reported to induce proliferation and differentiation of myeloid-lineage cells. Clinical trials of the independent usage of G-CSF or M-CSF showed better results than controls without G-CSF or M-CSF in chemotherapy and BMT (13-17). However, there is controversy concerning the benefits of using GM-CSF with leukemia patients, as described in the Introduction. Therefore, it was deemed appropriate to try the combination of G-CSF and M-CSF in anticipation of the synergistic effects on hematopoiesis. We showed that mice treated with a combination of G-CSF and M-CSF could form more CFU-S and developed a heavier spleen weight than control mice without cytokines in allogeneic BMT. These data are consistent with previous data, which indicated the effects of G-CSF and M-CSF (13-17). Moreover, there appeared to be a synergistic effect, augmenting CFU-S and spleen weight, when G-CSF and M-CSF were combined. The impact on the number of WBCs in the PB and number of nuclear cells in the BM was not as apparent as on 
CFU-S counts and spleen weights, but there were some effects. The number of donor cells also increased in the PB and the $\mathrm{BM}$ in cytokine-treated mice in comparison with control mice without cytokines. We have also shown a synergistic effect of G-CSF and M-CSF on augmentation of number of donorderived hematopoietic cells. It has been described that G-CSF accelerates the differentiation of bone marrow stem cells (BMSCs) into granulocytes and enhances the function of granulocytes, while M-CSF accelerates the differentiation of BMSCs into monocytes and enhances the function of monocytes and macrophages. Moreover, M-CSF can accelerate the proliferation and differentiation of stromal cells in the BM, which helps prepare an environment of hematopoiesis (18-20). It has been also reported that the G-CSF receptor does not have tyrosine-kinase activities in its intracytoplasmic region and that signal transduction from the G-CSF receptor uses Jack-STAT-dependent pathway for differentiation and proliferation of bone marrow cells (21), while the M-CSF receptor has tyrosine-kinase activities in its intracytoplasmic region $(22,23)$. Therefore, it is conceivable that the signal transduction pathways of G-CSF and M-CSF are different and that the mechanisms between those cytokines on hematopoiesis are somehow different. We therefore believe that the side effects of cytokines could be reduced, and the effect on hematopoiesis augmented by adopting of the combination of G-CSF and M-CSF. G-CSF and M-CSF are used clinically and are deemed to be safe. This suggests that we might be able to use G-CSF and M-CSF together safely and expect synergistic effects on hematopoiesis even in clinical usage.

In this paper, we have shown synergistic effects of G-CSF and M-CSF on the hematopoiesis of donor cells in allogeneic BMT. Combined use of G-CSF and M-CSF augmented the hematopoiesis of donor BMCs, resulting in an increase in CFU-S counts, increased spleen weights, and increased numbers of donor-derived hematopoietic cells in the peripheral blood and bone marrow.

\section{Acknowledgments}

We thank Professor H. Okabe (Osaka University, Osaka, Japan) for the donation of EGFP-transgenic mice. We also thank Ms. Y. Tokuyama, Ms. M. Murakami-Shinkawa and Ms. S. Miura for their expert technical assistance, and also Mr. Hilary Eastwick-Field and Ms. K. Ando for the preparation of this manuscript. This work was supported by grants from 'Gakunai Zyosei' in Kansai Medical University, a grant from the 'Haiteku Research Center' of the Ministry of Education, a grant from 'Millennium' of the Ministry of Education, Culture, Sports, Science and Technology, Grant-in-Aid for scientific research (B) 11470062, Grants-in-Aid for scientific research on priority areas (A) 10181225 and (A) 1162221, and Health and Labor Science research grants (Research on Human Genome, Tissue Engineering Food Biotechnology), a grant from the 'Science Frontier' program of the Ministry of Education, Culture, Sports, Science and Technology, a grant from the 'The 21st Century COE Program' of the Ministry of Education, Culture, Sports, Science and Technology, and also a grant from the Department of Transplantation for Regeneration Therapy (sponsored by Otsuka Pharmaceutical Co., Ltd.), a grant from the Molecular Medical Science Institute, Otsuka
Pharmaceutical Co., Ltd., as well as a grant from Japan Immunoresearch Laboratories Co., Ltd. (JIMRO).

\section{References}

1. Ohno R, Tomonaga M, Kobayashi T, et al: Effect of granulocyte colony-stimulating factor after intensive induction therapy in relapsed or refractory acute leukemia. N Engl J Med 323: 871-877, 1990.

2. Vellenga E, Ostapovicz D, O'Rourke B and Griffin JD: Effects of recombinant IL-3, GM-CSF, and G-CSF on proliferation of leukemic clonogenic cells in short-term and long-term cultures. Leukemia 1: 584-589, 1987.

3. Becker S, Warren MK and Haskill S: Colony-stimulating factor-induced monocyte survival and differentiation into macrophages in serum-free cultures. J Immunol 139: 3703-3709, 1987.

4. Sanda MG, Bolton E, Mule JJ and Rosenberg SA: In vivo administration of recombinant macrophage colony-stimulating factor induces macrophage-mediated antibody-dependent cytotoxicity of tumor cells. J Immunother 12: 132-137, 1992.

5. Pollard JW, Hunt JS, Wiktor-Jedrzejczak W and Stanley ER: A pregnancy defect in the osteopetrotic (op/op) mouse demonstrates the requirement for CSF-1 in female fertility. Dev Biol 148: 273-283, 1991 .

6. Nemunaitis J, Shannon-Dorcy K, Appelbaum FR, et al: Longterm follow-up of patients with invasive fungal disease who received adjunctive therapy with recombinant human macrophage colony-stimulating factor. Blood 82: 1422-1427, 1993.

7. Ohno R, Miyawaki S, Hatake K, et al: Human urinary macrophage colony-stimulating factor reduces the incidence and duration of febrile neutropenia and shortens the period required to finish three courses of intensive consolidation therapy in acute myeloid leukemia: a double-blind controlled study. J Clin Oncol 15: 2954-2965, 1997.

8. Rowe JM, Andersen JW, Mazza JJ, et al: A randomized placebocontrolled phase III study of granulocyte-macrophage colonystimulating factor in adult patients ( $>55-70$ years of age) with acute myelogenous leukemia: a study of the Eastern Cooperative Oncology Group (E1490). Blood 86: 457-462, 1995

9. Stone RM, Berg DT, George SL, et al: Granulocyte-macrophage colony-stimulating factor after initial chemotherapy for elderly patients with primary acute myelogenous leukemia. Cancer and Leukemia Group B. N Engl J Med 332: 1671-1677, 1995.

10. Zittoun R, Suciu S, Mandelli F, et al: Granulocyte-macrophage colony-stimulating factor associated with induction treatment of acute myelogenous leukemia: a randomized trial by the European Organization for Research and Treatment of Cancer Leukemia Cooperative Group. J Clin Oncol 14: 2150-2159, 1996.

11. Okabe M, Ikawa M, Kominami K, et al: 'Green mice' as a source of ubiquitous green cells. FEBS Lett 407: 313-319, 1997.

12. Till JE and McCulloch E: A direct measurement of the radiation sensitivity of normal bone marrow cells. Radiat Res 14: 213-222, 1961.

13. Bensinger W, Singer J, Appelbaum F, et al: Autologous transplantation with peripheral blood mononuclear cells collected after administration of recombinant granulocyte stimulating factor. Blood 81: 3158-3163, 1993.

14. Peters WP, Rosner G, Ross M, et al: Comparative effects of granulocyte-macrophage colony-stimulating factor (GM-CSF) and granulocyte colony-stimulating factor (G-CSF) on priming peripheral blood progenitor cells for use with autologous bone marrow after high-dose chemotherapy. Blood 81: 1709-1719, 1993.

15. Fu S and Liesveld J: Mobilization of hematopoietic stem cells. Blood Rev 14: 205-218, 2000.

16. Masaoka T, Motoyoshi K, Takaku F, et al: Administration of human urinary colony stimulating factor after bone marrow transplantation. Bone Marrow Transplant 3: 121-127, 1988.

17. Masaoka T, Shibata H, Ohno R, et al: Double-blind test of human urinary macrophage colony-stimulating factor for allogeneic and syngeneic bone marrow transplantation: effectiveness of treatment and 2-year follow-up for relapse of leukaemia. Br J Haematol 76: 501-505, 1990.

18. Deryugina EI, Ratnikov BI, Bourdon MA, Gilmore GL, Shadduck RK and Muller-Sieburg CE: Identification of a growth factor for primary murine stroma as macrophage colonystimulating factor. Blood 86: 2568-2578, 1995. 
19. Yamada M, Suzu S, Akaiwa E, et al: Properties of primary murine stroma induced by macrophage colony-stimulating factor. J Cell Physiol 173: 1-9, 1997.

20. Tanaka-Douzono M, Suzu S, Yamada M, et al: In vivo stimulatory effect of macrophage colony-stimulating factor on the number of stroma-initiating cells. J Cell Physiol 178: 267-273, 1999.

21. Avalos BR: The granulocyte colony-stimulating factor receptor and its role in disorders of granulopoiesis. Leuk Lymphoma 28: 265-273, 1998.
22. Bourette RP and Rohrschneider LR: Early events in M-CSF receptor signaling. Growth Factors 17: 155-166, 2000.

23. Alonso G, Koegl M, Mazurenko N and Courtneidge SA: Sequence requirements for binding of Src family tyrosine kinases to activated growth factor receptors. J Biol Chem 270: 9840-9848, 1995. 